\title{
Generalizations of Hamilton's rule applied to non-additive public goods games with random group size
}

\author{
James A. R. Marshall* \\ Behavioural and Evolutionary Theory Lab, Department of Computer Science, Kroto Research Institute, University of Sheffield, Sheffield, UK
}

\section{Edited by:}

Sasha Raoul Xola Dall, University of

Exeter, UK

\section{Reviewed by:}

Ryan L. Earley, University of

Alabama, USA

Lauren Johanna Nicole Brent,

University of Exeter, UK

David Queller, Washington

University in St. Louis, USA

*Correspondence:

James A. R. Marshall, Department

of Computer Science, Kroto

Research Institute, University of

Sheffield, Broad Lane, Sheffield

S3 $7 H Q$, UK

e-mail: james.marshall@

sheffield.ac.uk
Inclusive fitness theory has been described as being limited to certain special cases of social evolution. In particular some authors argue that the theory can only be applied to social interactions having additive fitness effects, and involving only pairs of individuals. This article takes an elegant formulation of non-additive public goods games from the literature, and shows how the two main generalizations of Hamilton's rule can be applied to such games when group sizes are random. In doing so inclusive fitness theory is thus applied to a very general class of social dilemmas, thereby providing further evidence for its generality. Interestingly, one of the two predominant versions of Hamilton's rule is found to be mathematically easier to apply to the scenario considered, despite both necessarily giving equivalent predictions.

Keywords: Queller's rule, non-additivity, public goods game, Prisoner's Dilemma, Snowdrift game, random group size

\section{INTRODUCTION}

Over the decades since its conception, inclusive fitness theory has been the subject of much misunderstanding. Two particular recurrent misunderstandings are considered in this article. First, that inclusive fitness theory cannot deal with non-additive fitness effects, which arise when the total fitness effect on social partners of their combined behavior is more, or less, than a simple sum of the fitness effects associated with those behaviors (e.g., van Veelen, 2009; Nowak et al., 2010; Traulsen, 2010). Second, that inclusive fitness theory can only deal with pairwise social interactions (e.g., Nowak et al., 2010). Hamilton's rule has, however, seen two main generalizations. Both of these are due to D. C. Queller, who originally extended Hamilton's rule with an additional term to account for non-additive fitnesses (Queller, 1985), and subsequently directly generalized Hamilton's rule in its original form by expressing fitnesses of types in terms of underlying genes rather than in terms of phenotypes (Queller, 1992a). This article has three main goals; first to generalize Queller's rule (Queller, 1985), following a hint in Queller (1992a), to deal with arbitrary interaction group sizes. Second, to apply both Queller's rule (Queller, 1985) and the generalization of Hamilton's rule (Queller, 1992a) to non-additive public-goods games (Motro, 1991; Hauert et al., 2006; Archetti and Scheuring, 2012) in groups of random size, thereby showing how inclusive fitness theory is applicable to pairwise additive interactions. Third and finally, to compare the mathematical tractability of the two alternative methods of incorporating non-additivity in inclusive fitness theory.

\section{HAMILTON'S RULE}

To re-introduce Hamilton's rule I shall use the Price equation approach to modeling selection of any kind (Price, 1970), and apply it to a public-goods game. The Price equation shows that the inter-generational response to selection on some trait, assuming faithful transmission on average, is given by

$$
\Delta \mathrm{E}(G) \propto \operatorname{Cov}(G, W)
$$

where $G$ and $W$ are random variables for, respectively, the value of the trait in question and individual fecundity, and $\Delta \mathrm{E}(G)$ is the change in expected value of the trait within the population from one generation to the next. Thus, the change in the expected value of the trait due to selection is proportional to the covariance between trait value and fecundity of individuals. Now consider a model of fecundity in a "public-goods" game, in which donators $(G=1)$ pay a cost $c$ to contribute a benefit $b$ which is divided equally among the $N$ members of their group, where $N$ is a random variable and so groups can be of different size. Allowing variable group size will allow a more general result, and address claims that inclusive fitness theory is only applicable to pairwise interactions (van Veelen, 2009; Nowak et al., 2010) (this assumption was also relaxed in Queller, 1992a; see also Marshall, 2010a, 2011; Gardner et al., 2011). In this public-goods game, non-donators $(G=0)$ pay no cost, but receive a share of any group benefits provided by donators in their group including themselves. Note that donation and non-donation phenotypes are unconditionally expressed. Then fecundity is

$$
W=\frac{G^{\prime}}{N} B-G C,
$$

where $G^{\prime}$ is the absolute frequency of donators in the group, excluding $G, B=b$ is the benefit of receiving aid, and 
$C=c-b / N$ is the cost of providing it. Capital letters $B$ and $C$ denote the benefit and cost required for Hamilton's rule, while lowercase $b$ and $c$ represent payoffs arising from interactions; as is well known, the two are not necessarily the same (Grafen, 2006). Since benefits are shared by their donors, note that donation in a public goods game is personally beneficial, and hence not altruistic, whenever $b / N>c$. If we substitute Equation (2) into Equation (1), then solve for when donation receives positive selection, assuming $C$ and $B$ are uncorrelated with $G$ and $G^{\prime}$, respectively, we obtain

$$
\left(\frac{\operatorname{Cov}\left(G, G^{\prime} / N\right)}{\operatorname{Var}(G)}\right) \mathrm{E}(B)-\mathrm{E}(C)>0,
$$

which is a version of Hamilton's rule (Hamilton, 1964, 1970), the classic result of inclusive fitness theory. In this version of Hamilton's rule relatedness (the covariance ratio in inequality 3) can be interpreted as a regression coefficient quantifying the degree of association between an individual's genetic trait, and the relative frequency of donators in their group, excluding themselves. Costs and benefits of donation are averaged across the population; in this case $\mathrm{E}(B)=b$ and $\mathrm{E}(C)=c-\mathrm{E}(b / N)$. Note that group size $N$ appears in the relatedness coefficient, rather than in the benefit $B$, since $G^{\prime}$ and $N$ are not generally independent, and hence cannot easily be separated in the covariance calculations.

It has been claimed that Hamilton's rule gives valid predictions only when fitnesses are additive (or when selection is weak) (Traulsen, 2010; van Veelen, 2009; Nowak et al., 2010). In fact, these are not new claims and they have been addressed previously in the literature, as reviewed most recently in Marshall (2011), Gardner et al. (2011). One approach, due to D. C. Queller, extends Hamilton's to deal with, among other refinements, non-additive interactions (Queller, 1985). Queller's original example of this was presented using a "synergistic" term for interactions in pairs, so fecundity becomes

$$
W=G^{*} b-G c+G G^{*} d,
$$

where $G^{*}$ is the genotype of the opponent ( 0 or 1 for non-donator or donator, respectively, just as for $G$ ), and $d$ is the deviation from additivity, with $d<0$ capturing diminishing returns from donation, $d>0$ capturing positively synergistic donation effects, and $d=0$ recovering additive donation. Equation (4) can be substituted into Equation (1) and re-arranged to give an extended version of Hamilton's rule (cf. Marshall, 2010b) showing that donation is favored when

$$
\left(\frac{\operatorname{Cov}\left(G, G^{*}\right)}{\operatorname{Var}(G)}\right) b-c+d \frac{\operatorname{Cov}\left(G, G G^{*}\right)}{\operatorname{Var}(G)}>0,
$$

where the left-hand-side of the inequality is the original regression formulation of genetic relatedness (e.g., Hamilton, 1970), and the right-hand side a modification of the familiar cost-benefit ratio from Hamilton's rule (Hamilton, 1964). However, as pointed out by Queller himself (Queller, 1985), the synergistic term and hence Queller's rule will have to change according to the size of interaction groups. Subsequently others have shown that if there are no restrictions on the payoff matrix, even for interactions with the same group size the rule could have to change from case to case (van Veelen, 2011). Both these points seem to limit the potential of such a rule as a general evolutionary principle, and appear especially problematic for the public-goods game we considered above with variable group sizes. Queller's rule has been generalized for larger group sizes, and applied to social evolution in bacteria (Smith et al., 2010). In the next section we shall see an alternative, very simple, generalization of Queller's rule that avoids the problems just identified.

\section{QUELLER'S RULE GENERALIZED}

By extending the original treatment of non-additivity (Queller, 1985) outlined in the previous section, we can see that there is in fact a general and concise inclusive-fitness rule for nonadditive interactions. To see this, we capture all deviations from interaction additivity in a single random variable $D$, which can depend on $G$ and $G^{\prime} / N$. This approach was mentioned in passing by Queller, when describing a general approach to dealing with correlated characters (Queller, 1992a). So fecundity in our original public-goods game is now extended from Equation (2) to be

$$
W=\frac{G^{\prime}}{N} B-G C+D
$$

Substituting Equation (6) into Equation (1) and rearranging into a similar form to inequality (3) gives

$$
\left(\frac{\operatorname{Cov}\left(G, G^{\prime} / N\right)}{\operatorname{Var}(G)}\right) \mathrm{E}(B)-\mathrm{E}(C)+\beta_{D G}>0,
$$

where $\beta_{D G}$ is the coefficient of a linear regression of $D$ on $G, \beta_{D G}=\operatorname{Cov}(G, D) / \operatorname{Var}(G)$, or in other words the extent to which individual type predicts receipt of non-additive payoffs (cf. Queller, 1992a), and similarly $\beta_{G^{\prime} / N, G}$ is the coefficient of the linear regression of $G^{\prime} / N$ on $G$, which is genetic relatedness as described above. Thus, inclusive fitness theory still admits a general rule dealing with non-additive interactions, regardless of interaction structure, by considering the sign and magnitude of the association between social traits and synergistic effects encountered, as captured in a single regression coefficient. Note that since $D$ is a random variable, it could incorporate any complexity in non-additive effects (cf. van Veelen, 2011) while allowing inequality 7 to remain valid. Note also that this version of Queller's rule is derived in terms of genes for unconditionally expressed traits, however an equivalent derivation in terms of conditionally-expressed phenotypes is also possible (Queller, $1992 \mathrm{a})$. In the case where focal individual's genetic value $(G)$ is used as the predictors in the regression $\beta_{D G}$, and generally, then regression theory guarantees that any residuals from the regression model will be uncorrelated with that predictor; this is important as it enables a quantitative genetics separation between selection coefficients and heritability coefficients (Queller, 1992b; Birch and Marshall, 2014). The generality given by the form of condition 7 comes at a cost however, if we are interested in causal explanations for evolutionary change 
(Marshall, 2015). In particular, although partners' genetic values are clearly causally-linked to receipt of non-additive fitness effects, the regression coefficient $\beta_{D G}$ does not include $G^{\prime} / N$ as a predictor.

As an example of the general applicability of Queller's rule (7), let us consider a formalization of the non-additive public-goods game for arbitrary group size (Hauert et al., 2006); based on earlier work (Motro, 1991) this formulation captures the major classes of social interaction, from the additive donation game, to the non-additive Prisoner's Dilemma and Snowdrift games (Hauert et al., 2006). In the notation of this letter, payoff for an individual in a group is defined as

$$
W=\frac{b\left(1-w^{G^{\prime}} w^{G}\right)}{N(1-w)}-G c
$$

where $w$ is a parameter controlling additivity, with $w<1$ leading to diminishing returns from additional donators, and $w>1$ leading to positive synergistic effects when multiple donators interact. Payoff additivity is recovered when $w=1$, as shown by application of l'Hôpital's rule to Equation (8) to give expected costs and benefits as occur in condition 3 (Marshall, 2015).

Let us add further detail to the non-additive model of Hauert et al. (2006) by considering arbitrarily-sized groups, formed by a process of aggregation. Specifically, let us consider groups that aggregate around individuals according to a geometric process with parameter $a$, so that an individual or group is joined by another individual with probability $a$, and with probability $1-a$ the group does not grow further. Each new member of a group could be one of two types, either donator or non-donator. Let us introduce an assortment parameter $\alpha$ that gives the probability that new members joining the group are of the same type as the founder member, while with probability $1-\alpha$ they are drawn according to the frequencies of the two types within the population. Once groups are formed, all individuals either donate to the public good or not according to their type, and the public good produced is shared equally amongst all group members. To analyse social evolution in this scenario we need to calculate all the component parts of inequality (7); this is done in Appendix B. Substituting these into inequality (7) gives conditions for donation to spread in the population under additive $(w=1)$ and non-additive $(w \neq 1)$ interactions, as described in Appendix B. Taking the additive case first, the condition for donation to receive positive selection is

$$
\begin{aligned}
& \left(\frac{\alpha(a-(a-1) \ln (1-a))}{a}\right) \\
& \quad b-(c+b(1-a) \ln (1-a) / a)>0 .
\end{aligned}
$$

The first coefficient in inequality (9) is the genetic relatedness coefficient of Hamilton's rule, multiplied by the expected benefit from donation $\mathrm{E}(B)=b$. By inspection, this relatedness coefficient is positive whenever $\alpha>0$ and $0<a<1$, since it is known that $a /(a-1)<\ln (1-a)$ for $0<a<1$. This has a natural interpretation, in that genetic relatedness in Hamilton's rule is positive for this scenario whenever there is a possibility for finite groups larger than 1 to form $(0<a<1)$, and there is positive assortment between the type of founding members and of joining members $(\alpha>0)$. Note that this genetic relatedness is that of the founder individual to the rest of the group. Relatedness within groups formed by aggregation is not necessarily the same between all members of the group. A general calculation of relatedness within such groups is substantially more complicated and is beyond the scope of the present article, but for illustrative purposes the simpler calculation presented is sufficient.

Considering the non-additive case next, as shown in Appendix B selection for donation occurs when (having moved to the equivalent, for altruism, $r>c / b$ form of Hamilton's rule)

$$
\begin{gathered}
\frac{\alpha(a-(a-1) \ln (1-a))}{a}>\frac{c+b(1-a) \ln (1-a) / a}{b} \\
-\frac{g-1}{a(1-g)}(a \alpha+(a-1+\alpha(1-a)) \ln (1-a) \\
(a-1)\left(\begin{array}{c}
\left.\frac{w \ln [1+a(\alpha(1-w)+g(\alpha-1)(w-1)-1)]}{\alpha(1-w)+g(\alpha-1)(w-1)-1}\right) \\
-\frac{\ln [1+a(g(\alpha-1)(w-1)-1)]}{g(\alpha-1)(w-1)-1}
\end{array}\right) .
\end{gathered}
$$

By comparison with inequality 9 , condition (10) is seen to be an extension of Hamilton's rule for the additive scenario, with the last term corresponding to (a simplification of) the synergistic term of Queller's generalized rule, that captures the deviation from payoff additivity occurring in this particular model $\left[-\beta_{D G} / \mathrm{E}(B)\right.$ in inequality 7$]$. Thus, the generalization of Queller's rule (7) is shown to be applicable to a very general class of social interactions, non-additive public-goods games with random group sizes; such interactions are of great biological relevance, since social behavior in groups of unpredictable size occurs very frequently, such as in the formation of stalk and fruiting body by Dictyostelium (Strassmann et al., 2000) or the production of siderophores by bacteria (West and Buckling, 2003), and diminishing returns from or synergy of social behavior are likely to be much more common than strictly additive interactions.

\section{HAMILTON'S RULE GENERALIZED}

Although, as demonstrated above, Queller's rule can be applied to non-additive interactions in groups of more than two, to some it may seem insufficiently "inclusive fitness-like," since it introduces an additional parameter beyond the original relatedness, cost and benefit of Hamilton's rule. However, Hamilton's rule can also incorporate non-additive interactions directly, without needing to be extended beyond these three fundamental parameters. This point was first made in Queller (1992a), which noted that Hamilton's rule fails to be accurate when applied to nonadditive interactions only when payoffs are expressed in terms of phenotypes, rather than the underlying genotypes. The solution is to construct a model of fecundity in terms of the underlying genotypes, using what Queller referred to as the "genic" model. This is achieved by deriving the costs and benefits in Hamilton's rule by, respectively, a partial regression of fecundity 
on an individual's genotype, and on those of their social partners (Queller, 1992a; Gardner et al., 2007, 2011). This yields an elegant version of Hamilton's rule with all its parameters being regression coefficients,

$$
\beta_{W G . G^{\prime} / N}+\beta_{\left(G^{\prime} / N\right) G} \beta_{W\left(G^{\prime} / N\right) . G}>0,
$$

where $\beta_{X Y . Z}$ is the partial regression of $X$ on $Y$, holding $Z$ constant (Queller, 1992a). Thus, the coefficients $\beta_{W G . G^{\prime} / N}$ and $\beta_{W\left(G^{\prime} / N\right) . G}$ correspond, respectively, to the cost to the actor of donation $(-c$ in Hamilton's original formulation) and the benefit to the recipient of donation ( $b$ in Hamilton's original formulation) (Queller, 1992a).

Condition 11 is a fully general version of Hamilton's rule for a public goods game. It does, however, have some potential shortcomings. First, there is no guarantee that the separation condition, which predicts when a decomposition of response to selection into selection and heritability coefficients can be achieved, will be satisfied (Queller, 1992a,b). Second, non-additive fitness effects are split between social partners (e.g., Gardner et al., 2011), which may raise issues in some eyes over the proper causal attribution of fitness effects. Leaving aside these issues, to illustrate the generality of inequality (11) we can apply it to the particular non-additive public-goods scenario with random group sizes presented above, just as it has already been applied to non-additive pairwise interactions (Gardner et al., 2007, 2011). This is achieved by taking a linear model of fecundity (Queller, 1992a; Gardner et al., 2007, 2011)

$$
\begin{aligned}
\hat{W}= & \mathrm{E}(W)+\beta_{W G \cdot G^{\prime} / N}(G-\mathrm{E}(G)) \\
& +\beta_{W\left(G^{\prime} / N\right) \cdot G}\left(G^{\prime} / N-\mathrm{E}\left(G^{\prime} / N\right)\right) .
\end{aligned}
$$

Note that using a linear model of fecundity does not assume that the relationship between fecundity and the genotypes of self and social partners is itself linear (Okasha, 2006; Gardner et al., 2011). Now, to derive the correct cost and benefit for Hamilton's rule, even in the presence of non-additive effects, we determine the partial regression coefficients that minimize the error of the model in predicting actual fecundities, with error defined as the sum of the squared residuals as for standard linear regression (Gardner et al., 2007, 2011). Thus, the error to be minimized is

$$
\mathrm{E}\left((\hat{W}-W)^{2}\right)
$$

Expression (13) is instantiated for the public-goods scenario described above in Appendix C. Unfortunately, a closed-form solution, and hence differentiation and minimization, of this appears to be intractable, as described in that same Appendix. Fortunately an alternative approach can be taken, calculating the partial regression coefficients directly from variances and covariances of the relevant random variables (e.g., Gardner et al., 2011). This is done in online Supplementary Material. The resulting partial regression expressions for cost and for benefit are very large and complicated, and hence are shown only in online Supplementary Material. However, substituting these and the regression definition of relatedness into condition 11 leads to the same answer as the Queller's rule approach (condition 10), as shown in online Supplementary Material.

\section{DISCUSSION}

This letter has shown how two inclusive-fitness-based rules for analysing social evolution can be applied to a model of non-additive public goods interactions in groups of varying and arbitrary size. This is useful on two counts. First, it has been observed that social interactions in groups of variable size have neglected by theorists, and that variance in group size needs to be taken account of in analysing social interactions within genetically unrelated groups (Peña, 2012). Second, the analyses presented here address criticisms that inclusive fitness cannot actually deal with non-additive interactions or interactions in groups larger than two which, although previously shown to be incorrect (as reviewed in Marshall, 2011 and Gardner et al., 2011) have recently resurfaced (Traulsen, 2010; van Veelen, 2009; Nowak et al., 2010). It has also been claimed that such rules must change from case to case (van Veelen, 2011), that these rules fail to separate "population structure" (i.e., relatedness, and trait frequencies) from "fitness effects" (i.e., costs and benefits of donation) (van Veelen, 2009), and that therefore inclusive fitness theory is of limited applicability (Nowak et al., 2011). The results of this letter illustrate how the generalized rules for social evolution need not change from case to case in anything other than their details; this is no different to how the details of any selective rule change according to the scenario they are applied to, whether that be the group-selection rule that donation spreads when between-group selection exceeds within-group selection, or even the general evolutionary rule that a trait spreads in a population when it is positively associated with reproduction. Regarding the separation of details of the population from the fitness effects of costs and benefits, it has been pointed out elsewhere that this is a general feature of models of frequency-dependent selection, rather than being peculiar to rules derived from inclusive fitness theory (Marshall, 2011).

It is of interest to conclude by considering the success of the two inclusive-fitness rules as applied to the scenario considered here. While both yield the same answer in this social evolutionary scenario the first of these, Queller's generalized extension of Hamilton's rule (Queller, 1992a) proves mathematically much easier to work with. In contrast, Hamilton's rule with fitness effects correctly defined in terms of genes rather than phenotypes (Queller, 1992a; Gardner et al., 2007, 2011) gives mathematically much more complicated expressions for the cost and benefit terms, and for still more complicated social scenarios it is reasonable to expect that it may prove mathematically intractable, while an approach using Queller's rule is still afforded. It is crucially important to note, however, that such a breakdown would not mean that Hamilton's rule is not a valid evolutionary principle for such a scenario, and therefore is not a general evolutionary principle; rather it would reflect the relative power of the mathematical techniques we can apply to such analyses. However, the results of this article do illustrate that an approach based on extending Hamilton's rule to deal separately with non-additive interaction effects can prove easier to apply, at least in some social scenarios. 


\section{ACKNOWLEDGMENTS}

I thank Geoff Wild and Corina Tarnita for comments on an earlier version of this manuscript.

\section{SUPPLEMENTARY MATERIAL}

The Supplementary Material for this article can be found online at: http://www.frontiersin.org/journal/10.3389/fevo.2014.00040/ abstract

\section{REFERENCES}

Archetti, M., and Scheuring, I. (2012). Review: game theory of public goods in one-shot social dilemmas without assortment. J. Theor. Biol. 299, 9-20. doi: 10.1016/j.jtbi.2011.06.018

Birch, J., and Marshall, J. A. R. (2014). Queller's separation condition explained and defended. Am. Nat. (in press).

Gardner, A., West, S. A., and Barton, N. H. (2007). The relation between multilocus population genetics and social evolution theory. Am. Nat. 169, 207-226. doi: $10.1086 / 510602$

Gardner, A., West, S. A., and Wild, G. (2011). The genetical theory of kin selection. J. Evol. Biol. 24, 1020-1043. doi: 10.1111/j.1420-9101.2011.02236.x

Grafen, A. (2006). Various remarks on Lehmann and Keller's article. J. Evol. Biol. 19, 1397-1399. doi: 10.1111/j.1420-9101.2006.01161.x

Hamilton, W. D. (1964). The genetical evolution of social behaviour I and II. J. Theor. Biol. 7, 1-52. doi: 10.1016/0022-5193(64)90038-4

Hamilton, W. D. (1970). Selfish and spiteful behaviour in an evolutionary model. Nature 228, 1218-1220. doi: 10.1038/2281218a0

Hauert, C., Michor, F., Nowak, M. A., and Doebeli, M. (2006). Synergy and discounting of cooperation in social dilemmas. J. Theor. Biol. 239, 195-202. doi: 10.1016/j.jtbi.2005.08.040

Marshall, J. A. R. (2010a). Queller's rule OK: comment on van veelen when inclusive fitness is right and when it can be wrong. J. Theor. Biol. 270, 185-188. doi: 10.1016/j.jtbi.2010.07.015

Marshall, J. A. R. (2010b). Ultimate causes and the evolution of altruism. Behav. Ecol. Sociobiol. 65, 503-512. doi: 10.1007/s00265-010-1110-1

Marshall, J. A. R. (2011). Group selection and kin selection: formally equivalent approaches. Trends Ecol. Evol. 26, 325-332. doi: 10.1016/j.tree.2011.04.008

Marshall, J. A. R. (2015). Social Evolution and Inclusive Fitness Theory: An Introduction. Princeton, NJ: Princeton University Press.

Motro, U. (1991). Co-operation and defection: playing the field and the ESS. J. Theor. Biol. 151, 145-154. doi: 10.1016/S0022-5193(05)80358-3

Nowak, M. A., Tarnita, C. E., and Wilson, E. O. (2010). The evolution of eusociality. Nature 466, 1057-1062. doi: 10.1038/nature09205

Nowak, M. A., Tarnita, C. E., and Wilson, E. O. (2011). Nowak et al. reply. Nature 471, E9-E10. doi: 10.1038/nature09836
Okasha, S. (2006). Evolution and the Levels of Selection. Oxford: Oxford University Press. doi: 10.1093/acprof:oso/9780199267972.001.0001

Peña, J. (2012). Group-size diversity in public goods games. Evolution 66, 623-636. doi: 10.1111/j.1558-5646.2011.01504.x

Price, G. R. (1970). Selection and covariance. Nature 227, 520-521. doi: $10.1038 / 227520 \mathrm{a} 0$

Queller, D. (1985). Kinship, reciprocity and synergism in the evolution of social behaviour. Nature 318, 366-367. doi: 10.1038/318366a0

Queller, D. C. (1992a). A general model for kin selection. Evolution 46, 376-380. doi: $10.2307 / 2409858$

Queller, D. C. (1992b). Quantitative genetics, inclusive fitness, and group selection. Am. Nat. 139, 540-558. doi: 10.1086/285343

Smith, J., Van Dyken, J. D., and Zee, P. C. (2010). A generalization of Hamilton's rule for the evolution of microbial cooperation. Science 328, 1700-1703. doi: $10.1126 /$ science. 1189675

Strassmann, J. E., Zhu, Y., and Queller, D. C. (2000). Altruism and social cheating in the social amoeba Dictyostelium discoideum. Nature 408, 965-967. doi: $10.1038 / 35050087$

Traulsen, A. (2010). Mathematics of kin- and group-selection: formally equivalent? Evolution 64, 316-323. doi: 10.1111/j.1558-5646.2009.00899.x

van Veelen, M. (2009). Group selection, kin selection, altruism and cooperation: when inclusive fitness is right and when it can be wrong. J. Theor. Biol. 259, 589-600. doi: 10.1016/j.jtbi.2009.04.019

van Veelen, M. (2011). A rule is not a rule if it changes from case to case: a reply to Marshall's comment. J. Theor. Biol. 270, 189-195. doi: 10.1016/j.jtbi.2010. 11.011

West, S. A., and Buckling, A. (2003). Cooperation, virulence and siderophore production in bacterial parasites. Proc. R. Soc. London B Biol. Sci. 270, 37-44. doi: $10.1098 / \mathrm{rspb} .2002 .2209$

Conflict of Interest Statement: The author declares that the research was conducted in the absence of any commercial or financial relationships that could be construed as a potential conflict of interest.

Received: 01 May 2014; accepted: 09 July 2014; published online: 29 July 2014. Citation: Marshall JAR (2014) Generalizations of Hamilton's rule applied to nonadditive public goods games with random group size. Front. Ecol. Evol. 2:40. doi: 10.3389/fevo.2014.00040

This article was submitted to Behavioral and Evolutionary Ecology, a section of the journal Frontiers in Ecology and Evolution.

Copyright $\odot 2014$ Marshall. This is an open-access article distributed under the terms of the Creative Commons Attribution License (CC BY). The use, distribution or reproduction in other forums is permitted, provided the original author $(s)$ or licensor are credited and that the original publication in this journal is cited, in accordance with accepted academic practice. No use, distribution or reproduction is permitted which does not comply with these terms. 


\section{APPENDIX A \\ METHODS}

Simplifications and solutions were performed with the symbolic algebra functions Simplify and Fullsimplify of Mathematica version 8.0.0.0 from Wolfram Research (see online Supplementary Material).

\section{APPENDIX B}

\section{QUELLER'S RULE FOR A NON-ADDITIVE PUBLIC-GOODS GAME}

Hauert et al. present an elegant formalization of non-additive public-goods games with arbitrary group size (Hauert et al., 2006). In the notation of this article, payoff for an individual in a group is defined by Equation (8).

We wish to express the deviation from payoff additivity in Equation (8) in terms of a single random variable $D$, as used in Equation (6), thereby separating additive from non-additive effects. This can be done by solving for equivalence between Equations (8) and (6), i.e., by solving

$$
\frac{b\left(1-w^{G+G^{\prime}}\right)}{N(1-w)}-G c=\frac{G^{\prime}}{N} B-G C+D
$$

for $D$, with $B=b$ and $C=c-b / N$ as described in the main text.

Now we model the process of group aggregation described in the main text. The (co)variances we need to calculate are $\operatorname{Var}(G), \operatorname{Cov}\left(G, G^{\prime} / N\right)$, and $\operatorname{Cov}(G, D)$. In general, $\operatorname{Cov}(X, Y)=\mathrm{E}(X Y)-\mathrm{E}(X) \mathrm{E}(Y)$. The expectations we require for $\operatorname{Cov}\left(G, G^{\prime} / N\right)$ can be calculated from the definitions of the geometric and binomial distribution as

$$
\begin{aligned}
& \mathrm{E}(G) \mathrm{E}\left(\frac{G^{\prime}}{N}\right)=g \sum_{n=0}^{\infty}\left((1-a) a^{n}\right) \\
& \quad\left(\frac{g \sum_{i=0}^{n} i\left(\begin{array}{l}
n \\
i
\end{array}\right)(g(1-\alpha)+\alpha)^{i}((1-g)(1-\alpha))^{n-i}}{n+1}\right. \\
& \left.+(1-g) \frac{\sum_{i=0}^{n} i\left(\begin{array}{l}
n \\
i
\end{array}\right)(g(1-\alpha))^{i}((1-g)(1-\alpha)+\alpha)^{n-i}}{n+1}\right),
\end{aligned}
$$

and

$$
\begin{aligned}
& \mathrm{E}\left(G \frac{G^{\prime}}{N}\right)=g \sum_{n=0}^{\infty}\left((1-a) a^{n}\right) \\
& \frac{\sum_{i=0}^{n} i\left(\begin{array}{l}
n \\
i
\end{array}\right)(g(1-\alpha)+\alpha)^{i}((1-g)(1-\alpha))^{n-i}}{n+1},
\end{aligned}
$$

while

$$
\mathrm{E}\left(G^{2}\right)=g
$$

and

$$
\mathrm{E}(G)^{2}=g^{2} .
$$

To calculate average cost of donation in the additive case

$$
\mathrm{E}(C)=c-\mathrm{E}\left(\frac{b}{N}\right)
$$

we also require the expectation of the reciprocal of group size

$$
\mathrm{E}\left(\frac{1}{N}\right)=-\frac{(1-a) \ln (1-a)}{a} .
$$

Substituting these into inequality (3) yields [provided $\operatorname{Var}(G) \neq$ 0 , i.e., $0<g<1$ ] the condition for donation to receive positive selection in an additive public-goods game, inequality 9 in the main text. Simplifications were performed with the symbolic algebra functions of Mathematica (Wolfram Research), as described in the Appendix; see online Supplementary Material.

Moving on to the non-additive public-goods game, as well as $\operatorname{Cov}\left(G, G^{\prime} / N\right)$ and $\operatorname{Var}(G)$, from the generalized version of Queller's rule (7) we now need to calculate

$$
\begin{aligned}
\operatorname{Cov}(G, D)= & \frac{b}{1-w}\left(\operatorname{Cov}\left(G, \frac{1}{N}\right)-\operatorname{Cov}\left(G, \frac{w^{G} w^{G^{\prime}}}{N}\right)\right) \\
& -b\left(\operatorname{Cov}\left(G, \frac{G}{N}\right)+\operatorname{Cov}\left(G, \frac{G^{\prime}}{N}\right)\right) .
\end{aligned}
$$

The first covariance in the rhs is zero, since $G$ and $N$ are independent in this scenario. Also, since $G$ and $N$ are independent, $\operatorname{Cov}(G, G / N)$ simplifies to $\operatorname{Var}(G) \mathrm{E}(1 / N)$. The expectations for the last covariance are given above in Equations (15) and (16). The relevant expectations for $\operatorname{Cov}\left(G, w^{G} w^{G^{\prime}} / N\right)$ are

$$
\begin{aligned}
& \mathrm{E}(G) \mathrm{E}\left(\frac{w^{G} w^{G^{\prime}}}{N}\right)=g \sum_{n=0}^{\infty}\left((1-a) a^{n}\right) \\
& \left(\frac{g \sum_{i=0}^{n}\left(\begin{array}{l}
n \\
i
\end{array}\right)(g(1-\alpha)+\alpha)^{i}((1-\alpha)(1-g))^{n-i} w^{i+1}}{n+1}\right. \\
& \left.+\frac{(1-g) \sum_{i=0}^{n}\left(\begin{array}{l}
n \\
i
\end{array}\right)(g(1-\alpha))^{i}((1-g)(1-\alpha)+\alpha)^{n-i} w^{i}}{n+1}\right)
\end{aligned}
$$

and

$$
\begin{aligned}
& \mathrm{E}\left(G \frac{w^{G} w^{G^{\prime}}}{N}\right)=g \sum_{n=0}^{\infty}\left(a^{n}(1-a)\right) \\
& \quad \frac{\left(\sum_{i=0}^{n}\left(\begin{array}{c}
n \\
i
\end{array}\right)((1-\alpha) g+\alpha)^{i}((1-\alpha)(1-g))^{n-i} w^{i+1}\right)}{n+1} .
\end{aligned}
$$

Substituting all of the above into inequality (7) and simplifying yields the condition for donation to receive positive selection, inequality (10) in the main text. 


\section{APPENDIX C}

\section{HAMILTON'S RULE FOR A NON-ADDITIVE PUBLIC-GOODS GAME}

As described in the main text, Hamilton's rule can also be directly applied to non-additive interactions, by correctly defining costs and benefits in terms of the partial regression coefficients minimizing the error of a linear model of fitness Equations $(12,13)$. For the particular scenario described in the main text, Equation (13) becomes

$$
\begin{aligned}
& \mathrm{E}\left((\hat{W}-W)^{2}\right)=\sum_{n=0}^{\infty}\left((1-a) a^{n}\right) \times \\
& \left(g \sum_{i=0}^{n}\left(\begin{array}{l}
n \\
i
\end{array}\right)(g(1-\alpha)+\alpha)^{i}((1-g)(1-\alpha))^{n-i} \times\right. \\
& \left(\mathrm{E}(W)+\beta_{W G . G^{\prime} / N}(1-g)+\beta_{W\left(G^{\prime} / N\right) . G}\right. \\
& \left.\left(\frac{i}{n+1}-\mathrm{E}\left(\frac{G^{\prime}}{N}\right)\right)+c-\frac{b\left(1-w^{i+1}\right)}{(n+1)(1-w)}\right)^{2}+ \\
& (1-g) \sum_{i=0}^{n}\left(\begin{array}{l}
n \\
i
\end{array}\right)(g(1-\alpha))^{i}((1-g)(1-\alpha)+\alpha)^{n-i} \times \\
& \left(\mathrm{E}(W)+\beta_{W G . G^{\prime} / N}(0-g)+\beta_{W\left(G^{\prime} / N\right) . G}\right. \\
& \left.\left.\left(\frac{i}{n+1}-\mathrm{E}\left(\frac{G^{\prime}}{N}\right)\right)-\frac{b\left(1-w^{i}\right)}{(n+1)(1-w)}\right)^{2}\right),
\end{aligned}
$$

where

$$
\begin{aligned}
\mathrm{E}(W) & =\sum_{n=0}^{\infty}\left((1-a) a^{n}\right) \\
& \left(g \sum_{i=0}^{n}\left(\begin{array}{l}
n \\
i
\end{array}\right)(g(1-\alpha)+\alpha)^{i}((1-g)(1-\alpha))^{n-i}\right. \\
& \left(\frac{b\left(1-w^{i+1}\right)}{(n+1)(1-w)}-c\right)
\end{aligned}
$$

$$
\begin{aligned}
& +(1-g) \sum_{i=0}^{n}\left(\begin{array}{l}
n \\
i
\end{array}\right)(g(1-\alpha))^{i}((1-g)(1-\alpha)+\alpha)^{n-i} \\
& \left.\frac{\left(b\left(1-w^{i}\right)\right)}{(n+1)(1-w)}\right)
\end{aligned}
$$

The standard procedure for linear regression is to differentiate an expression such as Equation (24) wrt to the partial regression coefficients, so that the minimizing values for them can be found, or to derive the regression coefficients directly from covariances and variances of the random variables concerned. Unfortunately since simplification to a closed-form solution of Equation (24) appears intractable (using the methods described in Appendix A no solution was found within a reasonable amount of time- $-30 \mathrm{~min}$ on a $2 \mathrm{GHz} 64$-bit Intel Core i5-equipped laptop-indicating either that the simplification is not possible or, if it is possible that the process and result of simplification are almost certainly much more complicated than the equivalent simplification for Queller's rule [resulting in Equation (10)], the former approach does not seem feasible in this particular case. Instead we use the fact that the partial regression coefficients of Equation (12) can be calculated directly from (co)variances (e.g., Gardner et al., 2011) as

$$
\beta_{W G . G^{\prime} / N}=\frac{\beta_{W G}-\beta_{W\left(G^{\prime} / N\right)} \beta_{\left(G^{\prime} / N\right) G}}{1-\rho_{G\left(G^{\prime} / N\right)}^{2}},
$$

and

$$
\beta_{W\left(G^{\prime} / N\right) . G}=\frac{\beta_{W\left(G^{\prime} / N\right)}-\beta_{W G} \beta_{G\left(G^{\prime} / N\right)}}{1-\rho_{G\left(G^{\prime} / N\right)}^{2}} .
$$

The above definitions are calculated in the online Supplementary Material for the non-additive public goods scenario in the main text, and substituted into condition 11 to give the same condition for selection of donation as that derived using the Queller's rule approach (10). 